\title{
Objective Quantification of Chest Radiography Exams for Pulmonary Fibrosis
}

\author{
G. Giacomini ${ }^{1}$, D.R. Pina ${ }^{2}$, M. Alvarez ${ }^{1}$, M. Oliveira ${ }^{1}$, A.L.M. Pavan ${ }^{1}$, S.M. Ribeiro ${ }^{2}$, P.C.M. Pereira ${ }^{2}$, \\ S.B. Duarte ${ }^{3}$, and J.R.A. Miranda ${ }^{1}$ \\ ${ }^{1}$ Dept. of Physics and Biophysics/Biosciences Institute of Botucatu, Univ. Estadual Paulista (UNESP), Botucatu, Brazil \\ ${ }^{2}$ Dept. of Tropical Diseases and Diagnosis Imaging/Botucatu Medical School, Univ. Estadual Paulista (UNESP), Botucatu, Brazil \\ ${ }^{3}$ Brazilian Center of Physics Research-CBPF-MCT, Rio de Janeiro, Brazil \\ \{matheus, jmiranda, analuiza\} @ibb.unesp.br, sbdecbpf.br, \\ \{marrone, ppereira, drpina\} afmb.unesp.br, marceladeoliveira@ig.com.br, \\ guigiacomini92@aluno.ibb.unesp.br
}

\begin{abstract}
Tuberculosis, caused by Mycobacterium tuberculosis, is an infectious disease which remains a global health problem. The chest radiography is the common method employed to assess the tuberculosis evolution. Despite the tuberculosis diagnosis be normally realized by chest radiography analyses, the objective quantification of pulmonary diseases has been developed with high resolution computed tomography scans. This quantification is important to assess evolution, treatment and comparison of different tuberculosis treatments. The aim of this work is to develop a methodology for pulmonary fibrosis quantification caused by tuberculosis using chest radiographs. An algorithm was developed in Matlab ${ }^{\mathbb{Q}}$ for computational processing of exams, which creates a lungs' $3 D$ representation with fibrosis regions. The developed method was performed with a group of 14 patients diagnosed with pulmonary Tuberculosis. For the same patients, the data were compared with quantification method of high resolution computed tomography scans, previously described in the literature. Statistical analysis was performed by linear regression and Bland-Altman plot. The results showed an average variation of $11.32 \%$ between the two quantification methods. The results suggest the effectiveness and applicability of the developed method, providing better riskbenefit to the patient and cost-benefit ratio for the institution.
\end{abstract}

Keywords - Pulmonary Fibrosis Quantification, Radiography, High Resolution Computed Tomography, Tuberculosis.

\section{INTRODUCTION}

Tuberculosis (TB) caused by Mycobacterium tuberculosis is an infectious disease which remains a global health problem [1]. It causes ill-health among millions of people each year and ranks as the second leading cause of death from an infectious disease worldwide, after the human immunodeficiency virus (HIV) [1]. In 2012, an estimated 8.6 million people developed TB and 1.3 million died from the disease [1]. While new treatments for TB are being developed, there are no suitable tools for quantifying the disease in both preclinical and clinical settings [2]. TB and other pulmonary diseases results in lung parenchyma inflammation and possibly ongoing fibrotic scar of the pulmonary interstitium and alveoli [3-4].

Chest radiography is the primary examination for TB diagnosis even in well-equipped medical center [5]. High
Resolution Computed Tomography (HRCT) scans are necessary when chest radiographs results are inconclusive for TB detection [6]. However, this method results in higher patient dose and more cost to the institution [7-8].

An objective quantification tool is of great importance for reliable and accurate detection of TB's pulmonary sequelae [2]. A reliable assessment assists the physician in monitoring the disease. Objective quantification is also important when comparing different treatments and drugs administration in order to evaluate their efficiency in a precise way [2;9]. Despite the TB diagnosis normally be realized by chest radiography analyses, the objective quantification of pulmonary diseases has been developed with HRCT scans [2-3;10-12], due to the high resolution power [13].

In this study, we developed an objective quantification method for determination of pulmonary fibrosis by chest radiographs, using TB as a disease model.

\section{Materials AND Methods}

This study was approved by the Research Ethics Committee of Botucatu Medical School, UNESP, under protocol CEP 008773/2013.

\section{A. Database}

This study analyzed 14 patients diagnosed with pulmonary TB and treated at the Infectious and Parasitic Diseases Service of the University Hospital, Botucatu Medical School, São Paulo State University - UNESP, Brazil, between January 2007 and May 2014. Gender distribution was predominantly males, with $13(92.86 \%)$ men. Mean age was $46.64 \pm 10.23$ years.

As inclusion factor, we selected patients with confirmed TB by bacilloscopy (BAAR), mycobacterium isolation in culture medium [14-15], for which HRCT and chest radiography exams had been obtained within a seven days interval. Patients with any other disease that could compromise the lungs, systemic diseases and aggravating factors except smoking and alcoholism were excluded. 


\section{B. Objective quantification method of chest radiographs exams for pulmonary fibrosis}

The objective quantification method for pulmonary fibrosis through chest radiography exams were implemented in Matlab ${ }^{\circledR}$ R2013a (Mathworks, Massachusetts U.S.A.). First, the lung was manually segmented from the X-rays exam by a radiologist in lateral and posterior-anterior (PA) views. The pulmonary fibrosis was segmented only in the PA view, since lateral view contains more superimposed structures [16].

The total lung volume was obtained by intersection of segmented lungs (PA and Lateral) sequentially expanded. The fibrosis thickness was estimated by a relation between the relative thickness of pulmonary fibrosis and Signal Difference to Noise Ratio (SDNR). The SDNR is measured by equation 1 [17], where $\mathrm{A}$ is the average pixel intensity of the fibrosis (detail), $\mathrm{B}$ and $\mathrm{C}$ are, respectively, the average pixel intensity and the standard deviation of the healthy lung (background).

$$
S D N R=\frac{|A-B|}{C}
$$

The relative fibrosis thickness was measured in HRCT scans while the SDNR was measured in PA view chest radiography for the same patient. Measurements were performed in the same region in both types of examination (Xray and HRCT), as illustrated in Figure 1. The red rectangle is the axial HRCT slice (xz plan), in which the relative thickness of fibrosis was measured. The green rectangle is the coronal slice (xy plan), obtained by software InVesalius 3.0, representing the PA view X-ray chest plan, in which the SDNR was measured.
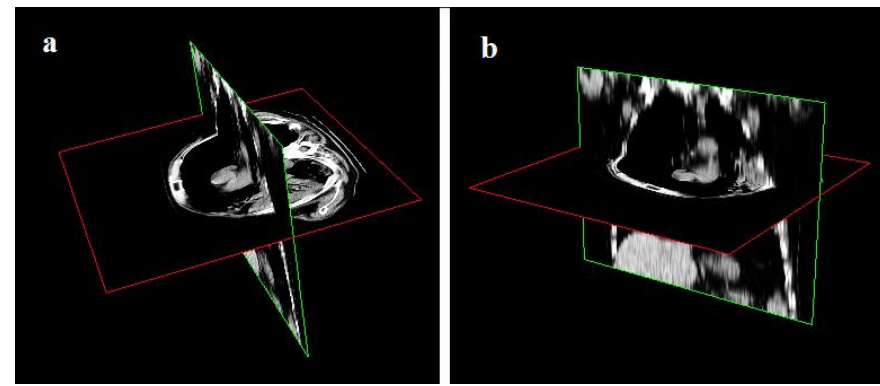

Fig. 1 Two views ( $a$ and $b$ ) of the axial slice (red) and coronal slice (green).

The obtained relation was used as a reference pattern for pulmonary fibrosis quantification, without the necessity of news HRCT scans. The SDNR in the X-ray examinations was used to estimate the relative fibrosis thickness. Then, a disk dilation process in the fibrosis area, previously seg- mented by a radiologist, got volume and was inserted in the total lung volume, as shows figure 2 . Hence, the objective quantification was calculated by the fibrosis and lungs volume ratio.

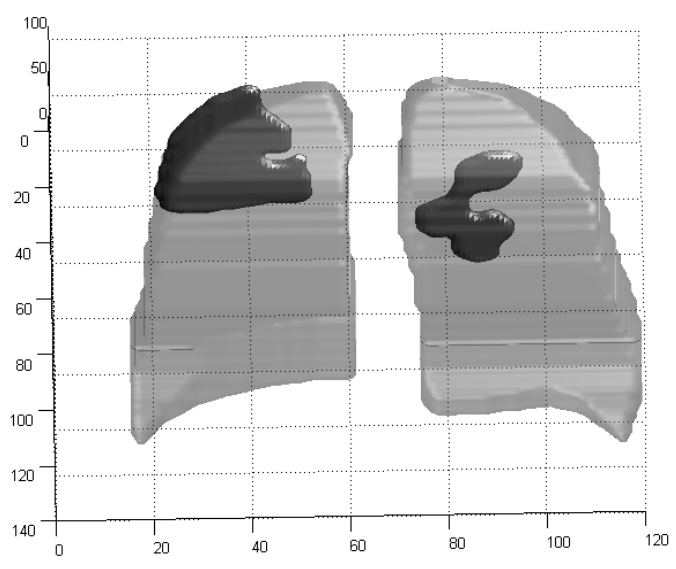

Fig. 2 Visualization of lungs and fibrosis.

The SDNR's behavior was assessed by a chest homogeneous phantom [18], with a step wedge [19] simulating different fibrosis thicknesses. The image was acquired with a technique commonly used in chest radiography routine $(90 \mathrm{kVp} ; 2.8 \mathrm{mAs})$. The SDNR values were evaluated for each step of the image.

\section{Objective quantification method of high resolution computed tomography scans for pulmonary fibrosis}

The quantification results by chest radiography described above were compared with the quantification through HRCT scans, for the same patients. This method followed a segmentation process described by Prionas et al in 2010 $[12 ; 20]$ based on Hounsfield Units (HU). A semiautomatic algorithm was developed using a thresholding technique by analyzing the slice histogram. Morphological operations were applied to reduce the false positive pixels. As a result, an automatic count of the healthy and fibrosis pixels allowed the objective quantification of pulmonary structures.

\section{Statistical Analysis}

Objective quantification method of HRCT scans was used as reference standards. Statistical linear regression and mean percentage difference were performed between data of both methods for the same patient. Repeatability (measurement of error) was determined as proposed by Bland and Altman [21]. 